TRAFFIC FLOW WIDE-AREA SURVEIL LANCE SYSTEM

\author{
Glenn O. Allgood \\ Regina K Ferrell \\ Stephen W. Kercel \\ Ruth A. Abston
}

Oak Ridge National Laboratory; Instrumentation and Controls Division

P.O. Box 2008, Oak Ridge, Tennessee 37831-6011

September 1994

To be presented at

SPIE International Symposium on

Photonics for Industrial Applications

Boston, Massachusetts

October 31-November 4, 1994

Managed by Martin Marietta Energy Systems, Inc., for the U.S. Department of Energy under contract DEAC05-84OR21400. 


\section{DISCLAIMER}

This report was prepared as an account of work sponsored by an agency of the United States Government. Neither the United States Government nor any agency thereof, nor any of their employees, make any warranty, express or implied, or assumes any legal liability or responsibility for the accuracy, completeness, or usefulness of any information, apparatus, product, or process disclosed, or represents that its use would not infringe privately owned rights. Reference herein to any specific commercial product, process, or service by trade name, trademark, manufacturer, or otherwise does not necessarily constitute or imply its endorsement, recommendation, or favoring by the United States Government or any agency thereof. The views and opinions of authors expressed herein do not necessarily state or reflect those of the United States Government or any agency thereof. 


\section{DISCLAIMER}

Portions of this document may be illegible in electronic image products. Images are produced from the best available original document. 


\title{
TRAFFIC FLOW WIDE-AREA SURVEILLANCE SYSTEM
}

\author{
Glenn O. Allgood \\ Regina K. Ferrell \\ Stephen W. Kercel \\ Ruth A. Abston \\ Oak Ridge National Laboratory, Instrumentation and Controls Division \\ P.O. Box 2008, Oak Ridge, Tennessee 37831-6011
}

\begin{abstract}
Traffic management can be thought of as a stochastic queuing process where the serving time at one of its control points is dynamically linked to the global traffic pattern, which is, in turn, dynamically linked to the control point. For this closed-loop system to be effective, the traffic management system must sense and interpret a large spatial projection of data originating from multiple sensor suites. This concept is the basis for the development of a Traffic Flow Wide-Area Surveillance (TFWAS) system. This paper presents the results of a study by Oak Ridge National Laboratory to define the operational specifications and characteristics, to determine the constraints, and to examine the state of technology of a TFWAS system in terms of traffic management and control. In doing so, the functions and attributes of a TFWAS system are mapped into an operational structure consistent with the Intelligent Vehicle Highway System (IVHS) concept and the existing highway infrastructure. This mapping includes identifying candidate sensor suites and establishing criteria, requirements, and performance measures by which these systems can be graded in their ability and practicality to meet the operational requirements of a TFWAS system. In light of this, issues such as system integration, applicable technologies, impact on traffic management and control, and public acceptance are addressed.
\end{abstract}

Keywords: wide-area surveillance, vehicle detection, traffic detection

\section{INTRODUCTION}

The Federal Highway Administration (FHWA) envisions an intelligent vehicle highway system of the future with sophisticated traffic management capabilities such as adaptive traffic flow control, automated route guidance, and platooning. These functions will require highly advanced surveillance systems that sense environmental and traffic conditions in order to characterize the state of traffic flow. ${ }^{1}$

Present-day traffic surveillance techniques typically employ point surveillance technologies that are inadequate for the needs of advanced future highway applications such as IVHS. Point surveillance can be likened to viewing a section of roadway, such as a turning lane, through an opaque black sheet with a hole cut in it. A point detector monitoring a turn lane may indicate the presence of a car waiting to turn. However, this detector alone cannot determine whether 1 or 20 cars are in the turn lane; nor can it indicate whether or not the car actually turned.

While more point detectors can be installed and the data from several point detectors can be integrated, the resulting information provides only an indication of traffic state in a limited area for a particular moment in time and is not sufficient for characterizing traffic flow. As the number of required point detectors increases, installation and maintenance costs escalate dramatically, rendering point detectors impractical for determination of traffic state. For future highway applications, surveillance of an entire roadway section from a broader viewpoint will be required. The concept of monitoring a larger section of roadway has been termed Traffic Flow Wide-Area Surveillance (TFWAS).

\footnotetext{
'Managed by Martin Marietta Energy Systems, Inc., for the U.S. Department of Energy under contract DEAC05-84OR21400.
} 
A TFWAS system will function as a transducer to the traffic control system; as a system it attempts to deduce the overall roadway flow pattern over a wide area and provide specific information to the control system. A wide area is defined as a geographic environment of finite extent within which attributes of ground vehicular traffic are monitored, processed, and managed to optimize the traffic movements with regard to throughput; safety; and environmental, economic, physical, and political constraints. A wide-area's dimensions might range in size from a tenth of a mile to several miles. A wide area will also be dynamically reconfigurable such that its extent may be adapted to traffic conditions. Additionally, this definition points out that the objective of the system is to optimize traffic flow with respect to certain constraints. A traffic flow pattern is the overall pattern of the flow of many vehicles, rather than the movements of individual vehicles. A traffic flow pattern reveals composite effects based on expectations and behavior patterns in a wide area.

For a human, this cognitive deduction of the traffic state is trained, volitional, and virtually an instantaneous process. The ultimate TFWAS concept is to emulate the process by which a human deduces the traffic conditions. It attempts to accomplish this task using a suite of sensors and cognitive algorithms to determine the traffic flow pattern. To design a system to perform the equivalent functional task is a very difficult undertaking. Under the sponsorship of the FHWA, Oak Ridge National Laboratory is involved in an effort to define the wide-area surveillance concept, to determine the constraints under which a system would operate, to examine the state of current technology in making such a system feasible, and to determine what must be done to make TFWAS successful in the long term.

\section{TFWAS FUNCTIONS AND ATTRIBUTES}

\section{Functions}

The primary function of a TFWAS system is to provide a description of traffic conditions to a traffic control system. This description includes both traffic data (velocities, density, queue lengths, etc.) and context data (time of day, weather conditions, etc.). It is envisioned that the specific attributes or structure of the description passed to the control system can be dynamically altered upon request by the control system.

The TFWAS system and the control system will also interact with a human-operated traffic management center (TMC). Thus, in addition to generating a machine-readable concept of the flow pattern, the TFWAS system must also be capable of generating a quickly comprehendible verbal/graphical interpretation of the flow pattern.

Because there are very few vehicles presently equipped with any special transponders and because some present vehicles can be expected to remain in service for 15-30 years, initial TFWAS system requirements are developed under the assumption that vehicles are totally passive participants in the traffic sensing process. Thus, initially the TFWAS system must perform its task without any participation from individual vehicles. It is anticipated that vehicles will gradually be equipped with intelligent sensing and response capability. In the early stages of TFWAS system development, TFWAS system feature descriptions could supply information to in-vehicle route guidance systems. This service is expected to have the desired effect of reducing overall congestion and creating a more favorable context in which to operate the traffic control system. However, because the TFWAS system feeds information into traffic controls eventually to be acted on by the traveler, the control action occurs over an extremely slow response time. This slow response is compounded by the fact that the driver may or may not act as recommended by the control system. In a later evolution of the traffic control system, the smart highway can be envisioned to directly control some movements of the smart car, such as by organizing platoons in real time. In this case, control actuations mean that the traffic control system has physical control of individual vehicles. The response time to control actions could be much shorter with such a system. This implementation is decades in the future, but the initial implementation of the TFWAS system and traffic control system needs to be upwardly compatible with this capability.

\subsection{Safety}

The safety objective of the TFWAS system is "first, do no harm." That is, the TFWAS system must not add risk from either a performance or a human user's standpoint. Within the purview of "first, do no harm," fail-safe 
considerations must be included, such as designing a traffic control signal that does not display green in all directions when it fails. It is intended and expected that by improving the continuity of traffic flow, traffic control will become safer than at present.

Safety of the overall system must be examined with respect to its relationship to traffic management and control systems. For example, what are the consequences of an inaccurate description of the state of traffic or of information gleaned from a malfunctioning sensor? What type of control decisions and actions are made as a result of information from the TFWAS system? Can these actions cause accidents or harm to system users? The consequences of inaccurate surveillance information may change dramatically as the control system evolves. The accuracy and reliability of the surveillance system become particularly important if the control system evolves to a future vision of directly controlling vehicles, managing platoons, etc. At each stage of evolution of the system, the safety analysis must be performed to assure a continuing level of safety.

\section{Reconfigurability}

FHWA anticipates that the traffic control system will have a dynamically reconfigurable architecture. It expects, but does not require, that the architecture be hierarchical. In addition, a practical implementation supporting reconfigurability requires that the system be modular. An essential attribute of TFWAS system design is reconfigurability. Three kinds of reconfigurability have been identified. The first is reconfigurability of the sensor suite: sensors are easy to plug and unplug. The second is reconfigurability of the hierarchy of control: sometimes multiple systems operate as one; other times they operate autonomously. Finally, a system might reconfigure both its control architecture and information flows in real time in response to a changing context.

A dynamically reconfigurable sensor interface should be modular. Hardware should be provided to interface a variety of sensors to a general input/output (I/O) data bus in the TFWAS system. Software drivers should also be provided for each interface along with an intelligent interface module that can determine what sensors are on the system. The design must be such that sensors can be conveniently added to or deleted from the system during operation, and the system should be able to automatically detect which sensors are not in service.

Reconfigurability of hierarchy of control implies that the boundaries of a wide area may expand or shrink under various traffic conditions in order to optimize an overall control strategy. Data flows from various sensor suites and information flows between cognitive modules must be able to be integrated to optimize traffic descriptions.

Reconfiguration of information flows in response to a changing context is a capability that adds a great deal of flexibility to the TFWAS system. Based on the traffic flow information received by the control system, if the control system determines that it needs a different set of measures or if the control system recognizes a need to alter the parameters of the wide area, a request is made to the TFWAS system. The TFWAS then restructures its traffic description to better meet the needs of the control system. For example, this reconfiguration might be triggered by an event such as a dramatic change in the inflow of traffic from a particular part of the wide area or after detection of an incident.

\section{Reliability}

The reliability of the system and its components is an issue. One measure of TFWAS system performance can be the percentage of time the data integration module and the individual sensors are available and doing work. As a means to improve this performance, the system should include incipient failure detection. It should monitor its subsystems and detect the trends that indicate that, even though a subsystem is still operating within acceptable limits, it may fail soon. This monitoring can improve the reliability of the system by allowing changeouts or bypasses before a failure occurs. It is also possible to reduce maintenance costs by indicating what parts of the system actually need maintenance. 
The modularity and reconfigurability of the system will allow it to route around subsystems that have either failed or are about to fail. When the technology matures, reconfigurability, combined with the robustness of the cognitive machinery (cognition algorithms may be able to compensate for inaccurate or incomplete sensor data), should preclude the need for a major backup system. Unless numerous failures occur, the system should not experience catastrophic failure.

\subsection{Expandability}

System implementation will begin by deploying small TFWAS systems that are expected to grow. I/O interfaces that are easy to expand and processing algorithms that are scalable will be required. The property of scalability means that the computational cost of an algorithm is roughly proportional to the amount of information it processes. An algorithm whose execution time grows factorially as a function of area may work perfectly for monitoring one intersection but fail miserably when expanded to monitor four intersections simultaneously.

Growth has another implication: the interconnectivity required to integrate small, local TFWAS systems into a cohesive system. Upward compatibility with dynamically reconfigurable information flow must be included in the system from the beginning. That is, when several TFWAS systems expand until they begin to merge with others, it should be an easy transition to integrate them into a single system when the context demands it, to operate them autonomously when the context demands it, and to switch back and forth as conditions change.

\section{TFWAS COMPONENTS AND STRUCTURE}

An individual TFWAS system may vary from site to site based on the physical attributes of the wide area, on the extremities of weather conditions under which the system must operate, and on the traffic knowledge requirements of the control system for the wide area. To meet these requirements, a whole series of TFWAS systems could be developed for each different kind of physical setting. A better way to meet these criteria would be to develop a hierarchical modular system that can be customized to a physical setting by judicious selection of modules. The structure of the TFWAS system and its essential attributes should meet a basic standard. The basic components of a TFWAS system as shown in Fig. 1 include a suite of sensors, a cognition/data integration module, an interface to the control system, and communications modules both internal and external to the TFWAS system. Human factors and systems integration are technologies that must be considered in the TFWAS system design.

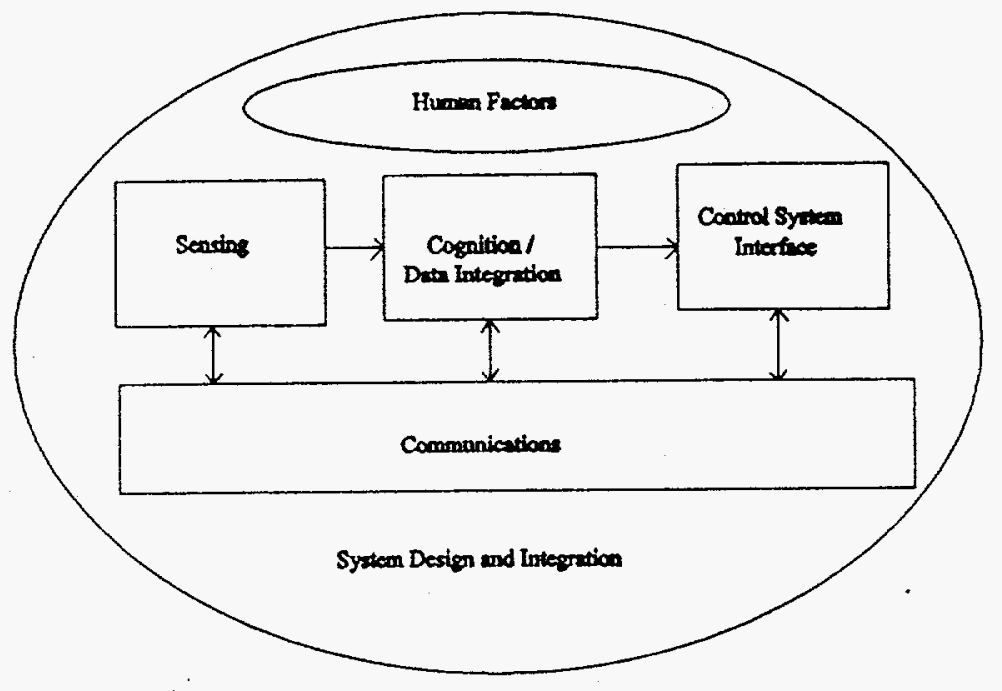

Figure 1. Major Technologies Involved in Creating a TFWAS 


\subsection{Sensing}

The sensor technologies most commonly used in the United States today are the inductive loop detector, the magnetic detector, and the magnetometer. ${ }^{2}$ These traditional technologies rely primarily on sensors buried in the pavement. Many newer technologies under development rely on above-the-road or roadside mounting. Although microwave radar detectors have been widely used for law enforcement, their use has been on a very limited basis for traffic detection. Also, ultrasonic detectors are widely used in Japan. Infrared sensors have been used for pedestrian crosswalks in England but are not used extensively for traffic detection. ${ }^{3}$ Video image processing for traffic control has been the subject of several research programs and has shown much promise in several studies. Concepts such as the use of probe vehicles and automatic vehicle identification (AVI) seek to take advantage of information or techniques that may not be practical or cost-effective to use solely in support of the TFWAS system but may exist in support of other aspects of the IVHS, such as toll collection or commercial vehicle operations.

Many factors should be considered when selecting sensors for a TFWAS system. Sensors selected must be able to operate in the environmental extremes in which the surveillance system functions. Environmental problems include the more obvious temperature extremes, rain, snow, sleet, and fog. Additional environmental hazards include wind, vibrations, shadowing, electromagnetic interference, and surrounding infrastructure, both manmade and natural. ${ }^{4}$ Sensors selected must have sensing ranges appropriate for the application and be capable of providing necessary traffic measures for the given application.

The sensors must be capable of installation within the highway infrastructure in a configuration that allows safe, reliable operation. Sensors should be selected to provide the most complete, accurate information about the traffic flow in the most cost-effective manner. Important cost considerations include sensor cost, installation cost, maintenance cost, life expectancy, and repair and replacement costs. The possibility (real or perceived) of the sensor causing injury to humans or damage to the environment is another important consideration.

Although several technologies are capable of sensing various traffic parameters, no single technology stands out as the lone provider of wide-area sensing capabilities in all settings and under all environmental conditions. By defining a TFWAS system architecture capable of supporting a multiple sensor technology suite, a robust system can be designed to best assess the traffic conditions for a particular setting while withstanding the environmental variations peculiar to that geographic area. This design philosophy also enables an evolutionary approach to traffic detection and characterization that can take advantage of future technological advances in any sensor category.

As part of this project, an extensive survey of conventional sensor technologies and emerging sensor technologies was conducted. These technologies were analyzed to determine their strengths and weaknesses as future TFWAS system components. This information is detailed in ORNLTM-12827, entitled Traffic Flow Wide-Area Surveillance Systems Definition.

\subsection{Data integration/cognition}

A fully implemented TFWAS system would use broadly based cognitive algorithms to discern various attributes that characterize the traffic flow pattern. The application of machine cognition to the problem of TFWAS must go beyond short-term applications. The National Program Plan for IVHS recognizes that in the full-featured wide-area surveillance system (required as the transducer into a full-featured adaptive traffic control system in a fully implemented IVHS), the major unsolved problem is that of machine cognition. ${ }^{1}$ How does the surveillance system take a large set of data flows and, from those, determine the pattern of traffic flow through the area under surveillance?

In the short term, existing data integration algorithms represent a straightforward solution to an easier problem. Data fusion algorithms exist that make no pretense at being cognitive algorithms. ${ }^{6}$ Through the judicious use of fusion algorithms, running on progressively faster and cheaper computers, dramatic improvements can be made in the ability of the present generation of "wide-area surveillance" systems to extract the maximum amount of information from the data being furnished by their sensor array. However, while presently available data fusion algorithms represent 
a means of improving the percepts delivered by the present generation of sensors, they will not present a conceptual view of traffic flow.

A number of recent developments of special cases could lead to improved processing of highway sensor data. These include incident detection using fuzzy set theory, incident detection using a Kalman predictor, ${ }^{8}$ traffic volume and classification monitoring, ${ }^{9}$ link travel times from point detector readings, ${ }^{10}$ and start-stop waves using cellular automata."

Specific algorithms have been developed to perform limited functions in precisely defined contexts. In a general purpose cognition algorithm, these functions would become special cases. The full-featured TFWAS of one or two generations in the future will need to provide a conceptual view of the traffic flow pattern. Present research in artificial intelligence has been investigating machine intelligence along four different paths. ${ }^{12}$ The one that seems the most promising for TFWAS is the inductive paradigm in which the machine learns a concept from repeated exposure to instances of, and counterexamples to, the concept. This is somewhat akin to the idea of an unsupervised pattern recognition algorithm, where given a sufficient number of instances, the algorithm recognizes the natural clusters that they form. As the TFWAS is continually exposed to instances of traffic flow, it is expected to draw conclusions from what is sees with virtually no feedback as to whether or not it got the right answer.

\subsection{Communications}

A TFWAS system might include hundreds of sensors in an area with dimensions ranging from a tenth of a mile up to several hundred miles. Raw or fused data from these sensors must be communicated to the processor that deduces the traffic flow characteristics. In addition to the need for localized communications within a TFWAS system, it will also be necessary to provide longer distance communications between collaborating systems.

A wide variety of communications techniques are available for use with a TFWAS system. These communications techniques can be broken down into two categories: land-lines and wireless. Land-lines include the low-bandwidth twisted pair and the higher bandwidth coaxial cable and fiber optics. Data may also be communicated in a wireless fashion using radio waves. These waves may be transmitted from point to point by satellite, meteor burst, or a series of terrestrial microwave transponders. Radio waves may also be transmitted to and from mobile transponders using satellites or terrestrial antennae. Narrowband or spread spectrum technology can be used. Commercially provided services such as cellular or packet radio can be used to take advantage of the existing communications infrastructures.

The selection of the technique or techniques used for a communications function will likely be application specific. The communications link must support the required data rate and must be appropriate for transmitting the type of data desired. Another consideration is whether transmissions occur continuously in real time or only intermittently. The distance over which data must be transmitted is important for cost comparisons. The existing infrastructure must be considered as well. Important cost and installation considerations include the availability of right-of-way for land-line installation, availability of frequency allocations for wireless communications, and the availability of existing commercial communications infrastructures that would provide adequate communications services. Important communications concerns include the possibility of interference from surrounding active devices, as well as possible environmental and biological effects. The interconnection scheme must also be considered. Are the media supporting a point-to-point communication of data, or are they part of a network of a distributed database? The communication technique chosen must be adaptable for future plans. Is future expansion anticipated and are the media of choice easily expandable? What operational life span is required? A final concern is the issue of security of the signal from interception or interference. A survey of communications technologies is included in ORNL/TM-12827. ${ }^{3}$

\section{MEASURES OF PERFORMANCE}

Several measures of performance will be applied to assess the performance of a TFWAS system, both on a global level and at a systems level. On a global level, the overall effectiveness of a TFWAS system will be assessed by 
how the operation of such a system changes measurable traffic flow parameters. At a systems level, the performance of the TFWAS system will be assessed relative to how well the TFWAS system measures and communicates data. The individual components of a TFWAS system will be assessed as well, with various parameters used to measure the performance of the sensor suites, the communications, and the data integration or cognition algorithms.

\subsection{Overall measures of performance}

Since the TFWAS system may be thought of as a transducer to the traffic flow control system, many of the same overall measures of performance used to assess the traffic flow control system may be used to assess a TFWAS system. The objective of a traffic flow control system is to simultaneously minimize delay and maximize throughput, while equalizing the traffic load. A secondary objective is to reduce energy consumption and improve air quality. It is likely that such parameters as link travel time, vehicle miles travelled, and slug velocity and acceleration will be measured and used to assess the performance of a TFWAS system.

Link travel time is defined as the average time for a vehicle to traverse a link in the transportation network. Link travel time is associated with delay but is a much easier parameter to define, assess, and control. Link travel time is very important both as a control parameter and as a planning tool because it provides quick feedback to assess the effect of a control action. It serves as a measure of congestion and can be used for a performance comparison of different links to identify critical links. Link travel time also serves as a quantitative measure of improvement due to new construction. ${ }^{10}$

Another important measure of performance is vehicle miles travelled. The improvement or degradation of a road system can be assessed by observing changes in the vehicle miles travelled per unit time. This parameter is also important to transportation planners who intend to double the vehicle miles travelled over the next 30 years with no increase in road capacity. ${ }^{1}$

The determination of slug velocity and acceleration as functions of flow may also be useful measures of performance. A slug velocity is the average velocity of a group of vehicles traveling in a cluster. Cycles in the slug acceleration would indicate stops and starts and would help pinpoint trouble spots.

Since it is an objective of traffic control to reduce energy use and improve air quality, the TFWAS system is expected to make some environmental measurements. Such environmental monitoring, while not used directly in the control function, can provide information on the overall effectiveness of the system. While air quality can be monitored, it is unlikely that fuel consumption can be measured directly. At best, a TFWAS system would be able to deduce some indication of fuel consumption from its observations of selected pollutant levels such as $\mathrm{CO}, \mathrm{NO}_{\mathrm{v}}$ lead, and hydrocarbon particulates.

While these parameters may not seem like measures of performance, they are important information the TFWAS system should determine and provide to the control system. They can be measures of the effectiveness of the control system and, indirectly, a measure of the quality of the information the TFWAS system provides.

\subsection{Systems level measures of performance}

At the systems level, the performance of a specific surveillance system is a measure of how well the system provides data. The measures of a system include reliability, reproducibility, accuracy, and repeatability. Repeatability refers to the system's ability to provide the same output for repeated instances of a given input. Reproducibility is associated with repeatability. Reproducibility is defined as the ability of different units to provide the same output for a given input. Accuracy is a measure of the deviation of the result from the true value. Reliability is a factor that strongly affects the availability of the system. Reliability includes such things as mean time between failures and percent downtime. 
The TFWAS system performs only as well as its components perform; therefore, the sensor suites, communications equipment, and processing algorithms should be assessed by measures of performance as well. While a vast variety of sensors can be used in a TFWAS system, some general performance indicators may be applied to all sensors. These are reliability, reproducibility, accuracy, and repeatability.

Error-free throughput is perhaps the most important measure of performance of a communications link. This indicates whether a communication method is suitable for the data transmission requirements. Error rate in number of erroneous bits per million bits decoded is the best indicator of overall utility of the link. Availability, another important measure, is a measure of the percent of time the link is available. This provides guidance to maintenance and indicates the need to provide redundant links.

Processing algorithms will be used broadly by the TFWAS system to discern various attributes that characterize the traffic flow pattern. Currently in use are specific algorithms to perform limited perceptive functions for a set of precisely defined circumstances. Cognitive algorithms for the TFWAS system application need to be developed since no such algorithms presently exist. The primary measure of performance of a processing algorithm is accuracy. Did the algorithm deduce the correct conclusion from the data presented to it? Another key measure of performance is time required to turn the stream of sensor percepts into a concept of overall traffic flow. Scalability is another important parameter; it measures the utility of the algorithm in terms of the complexity and size of system it can work with. A final measure of performance of the machine cognition algorithms is believability of the concepts it produces. Believability may not be directly measurable; however, there will likely be some indication of the acceptance of a particular algorithm.

\section{BARRIERS TO DEPLOYMENT}

Three of the biggest barriers to deployment of wide-area surveillance systems are costs, safety/liability issues, and privacy issues. These concerns cannot be addressed from a technical standpoint alone. The public perception of the system and the way these issues are addressed will have a major impact on TFWAS system deployment and on the system's effectiveness.

Three issues need to be addressed. First, "Is the public getting value for its money?" The answer depends on the capability of the TFWAS system and traffic control to make it noticeably easier for travelers to reach their destinations. "Is the system perceived as safe?" This issue is the most difficult to resolve. "Is privacy being safeguarded?" The answer depends on using a system that tracks aggregate flow patterns but not individual vehicles.

\subsection{Costs}

Several aspects of cost are to be considered in any large system. The capital equipment cost, installation cost, maintenance cost, operation cost, and the composite life cycle costs are all important considerations in selecting and implementing a wide-area surveillance system. Although estimating the life cycle cost may be practical and seem to be the best measure of a system, other cost considerations may be equally important from a funding viewpoint. The benefit of a TFWAS system may be difficult to estimate. An improved throughput is hard to quantify in terms of dollars, especially if a larger volume of traffic begins to use the roadway under a control system utilizing TFWAS. Even more difficult may be determining the value of the incremental increase in benefit of a more sophisticated TFWAS implementation than a simpler, less costly one.

\subsection{Safety and liability}

Who is responsible for damages (real and imagined) resulting from improper operation of the system? Is there a limit on liability? Is the risk of liability so great that the system is impractical to deploy? These and other issues will need to be settled by legislation. Many of the safety concerns for a wide-area surveillance system are also shared by other aspects of IVHS improvements. Thomas Sheridan pointed out several major safety problems with regard to 
IVHS..$^{13}$ Improved throughput may lead to an increase in volume and/or a decrease in safety. Low speeds are safer than high speeds, with the ultimate in safety occurring when no one is moving. People also tend to adjust their behavior to compensate for gains provided by automation. For example, people drive faster on wide, divided highways or follow more closely at higher speeds with improved braking systems on their vehicles.

Safety issues may arise from the individual components of the system. A major concern may be emission levels from active sensing or communication devices. A study of the vulnerability of humans to the energies radiated by IVHS sensors is an important action in addressing this concern. Scientific proof that the emission levels are safe, combined with a competent public relations effort, may go a long way toward allaying public fears.

\subsection{Privacy and ownership}

While wide-area surveillance systems were conceived for the role of optimizing traffic flow, providing information for routing services, and providing information to transportation planners, there are other possible uses for the information gathered. It has been suggested that the information would be useful for law enforcement activities or to raise revenue by selling the information to the public. There are some compelling reasons for not using TFWAS system outputs for anything but traffic flow control, route guidance, and transportation planning. First, to have the system sell information to the public could lead to litigation over who owns the information, what rights are conveyed by ownership, invasion of privacy, and liability for damage due to disclosure (accurate or inaccurate) of information generated by the TFWAS system. Deployment of the system could be delayed for years as these battles are fought through the courts. Part of the solution to this problem is to configure the system so that it does not identify or track individual vehicles. A second part of the solution is to include administrative and security procedures that do not provide the information to anyone but transportation officials.

Similarly, the use of TFWAS system information by law enforcement services could lead to several problems. To begin with, innocence is presumed under law. The use of electronic surveillance for traffic enforcement in the absence of human presence and verification is interpreted by many as violating the right to privacy implied by the Fourth Amendment to the U.S. Constitution. Because of the constitutional presumption of innocence and the requirement for proof beyond a reasonable doubt, the technical requirements for a law enforcement surveillance system are different from those of a traffic flow control system. A cognitive surveillance system can integrate poor-quality percepts into a flow concept that is adequate for efficient and safe traffic flow control. For law enforcement, highquality percepts would be required to provide compelling proof of guilt.

\subsection{Jurisdiction}

For optimal traffic control operations, the definition of some wide areas may cross county lines or include federal and state roadways. Each jurisdictional entity has differing budgets, priorities, and goals. While systems can be designed totally within single jurisdictions, cooperation among such groups will often be necessary to ensure optimization of resources and traffic flow.

\subsection{Infusion of new technology}

Although several new technologies are being developed for traffic sensing systems, most commercial products brought to market using these alternative sensors function to perform detection and measurement as an inductive loop or as multiple inductive loops. That is, these systems seek to emulate the use of inductive loops to provide traffic information. With the exception of a few video image processing systems that employ tracking strategies, true surveillance of entire areas is not being accomplished. This may be the result of supply and demand. Since control strategies have been adapted to the standard of the inductive loop, there is no real demand for a true wide-area surveillance function. Similarly, since there is not a clear understanding of what kind of real-time information can be made available to the control system, control strategies have not been developed that can take advantage of such information. 


\section{SUMMARY}

In the near term, wide-area surveillance technology will develop as driven by the market, independent of FWHA research priorities. Future advances will probably include the expansion into traffic control of sensor technologies previously developed as solutions for other problems and the increased use of data fusion algorithms to maximize the efficiency of existing sensor suites. These will result in improved performance as compared with existing wide-area surveillance systems, a larger area monitored, more accurate data, etc. The sensor technologies are either mature or are being aggressively developed. The problem for the future is what to do with the sensor data. The draft National Program Plan for $I V H S^{1}$ recognizes that for a full-featured wide-area surveillance system, required as the transducer into a full-featured adaptive traffic control system, in a fully implemented IVHS, the major unsolved problem is the problem of machine cognition. How does the surveillance system take a large set of data flows and, from those, conceptualize the pattern of traffic flow through the area under surveillance?

TFWAS has the potential to revolutionize traffic control strategies. TFWAS is designed to provide a global view of the overall state of traffic. Most present schemes are based on very limited knowledge of traffic conditions. Compared to present technology, this global traffic view provides more complete information for anticipatory control. TFWAS provides quick feedback on the overall effectiveness of a particular control action. An intelligent TFWAS system would reduce the burden on the control system by deducing flow parameters.

Wide-area surveillance is a concept that can take advantage of the strengths of different traffic sensing technologies, can readily adapt to newly developed technologies, and can grow with the development of new traffic control strategies. By developing sensors that can fuse information from multiple spectra and by developing algorithms that will take information from a variety of sensor types and develop descriptions of traffic flows over a wide area, a more comprehensive understanding of the traffic state can be provided to the control system to perform optimal realtime adaptive control actions over the entire wide area.

\section{ACKNOWLEDGMENTS}

This research is sponsored by the Department of Transportation's Federal Highway Administration Office of Safety and Traffic Operations Research and Development. Special thanks to Philip Moynihan of Jet Propulsion Laboratory for his thoughtful comments on the concept of wide-area surveillance, his review of this document, and his assistance in the latter stages of this project.

\section{REFERENCES}

1. National Program Plan for Intelligent Vehicle-Highway Systems, Draft, available from IVHS America, Washington, DC, May 1994.

2. Traffic Detector Handbook, 2nd ed., p. 2, JHK and Associates, PB91-164228, July 1990.

3. Traffic Detector Handbook, 2nd ed., p. 166, JHK and Associates, PB91-164228, July 1990.

4. Hughes Aircraft Corporation and JHK \& Associates, Vehicle Detector Field Test Specifications and Field Test Plan, Task Report F for Detection Technology for IVHS Contract Number DTFH61-91-C-00076, January 1994.

5. G. O. Allgood, R. K. Ferrell, S. W. Kercel, R. A. Abston, and P. I. Moynihan, Traffic Flow Wide-Area Surveillance System Definition, ORNL/TM-12827, Oak Ridge Natl. Lab., October 1994.

6. Lawrence A. Klein, Sensor and Data Fusion Concepts and Applications, SPIE Optical Engineering Press, Bellingham, WA, Tutorial Texts in Optical Engineering, Vol. TT14, 1993.

7. Edmond Chin-Ping Chang and Su-Ha Wang, "Improved Freeway Incident Detection Using Fuzzy Set Theory," 73rd Annual Transportation Research Board Meeting, Paper No. 940603, Washington, DC, Transportation Research Board, 1994.

8. D. J. Dailey, An Optimal Recursive Estimator for Detecting Traffic Anomalies Using Real Time Inductance Loop Data," 73rd Annual Transportation Research Board Meeting, Paper No. 940424, Washington, DC, Transportation Research Board, 1994. 
9. William C. Mead, Henry N. Fisher, Roger D. Jones, Keith R. Bisset, and Leopold A. Lee, "Application of Adaptive and Neural Network Computational Techniques to Traffic Volume and Classification Monitoring," 73rd Annual Transportation Research Board Meeting, Paper No. 940735, Washington, DC, Transportation Research Board, 1994.

10. Virginia Sisiopiku and Nagui M. Rouphail, "Towards the Use of Detector Output for Arterial Link Travel Time Estimation: A Literature Review," 73rd Annual Transportation Research Board Meeting, Paper No. 940160, Washington, DC, Transportation Research Board, 1994.

11. Kai Nagel and Michael Schreckenberg, "A Cellular Automaton Model for Freeway Traffic," J. Phys. I, pp. 2221-29 (December 1992).

12. Jaime Carbonell, Machine Learning, MIT Press, 1992.

13. Thomas B. Sheridan, "Safety and Human Factors in IVHS," IVHS Rev. (Winter/Spring 1994). 